\title{
Deep CCD photometry and the initial mass function of the core of the OB cluster Berkeley 86$^{\star}$
}

\author{
H.J. Deeg ${ }^{1,2}$ and Z. Ninkov ${ }^{1}$ \\ 1 Rochester Institute of Technology, Center for Imaging Science, Rochester, NY 14623, U.S.A. \\ 2 Instituto de Astrofisica de Canarias, Via Lactea s/n, 38200 La Laguna, Tenerife, Spain \\ email: hdeeg@iac.es, zxnpci@cis.rit.edu
}

Received August 17, 1995; accepted March 6, 1996

\begin{abstract}
Based on photometry of deep CCD frames of the central region of the OB cluster Berkeley 86 , we derive the cluster mass function. The absence of current star formation, and the cluster's young age of about 6 Myrs, leads to the conclusion that the initial mass function (IMF) and the current mass function are identical for stars with $m<10 m_{\odot}$. In the range of $1.2-20 m_{\odot}$, an IMF with a slope of $\Gamma=-1.3 \pm 0.3$ is found. This value agrees well with other recent determinations of young clusters IMFs which are close to the classical Salpeter IMF with $\Gamma=-1.35$. Sections of the IMF of Berkeley 86 that are significantly steeper, or flatter, are most likely the result of a dip in the star's mass distribution in the range of 3.5-10 $m_{\odot}$. Similar dips may have led to steep IMFs over narrow mass ranges, as reported in the literature for some other clusters. No sign for a low mass turn-over in the IMF of Berkeley 86 is found for masses extending down to $0.85 m_{\odot}$.
\end{abstract}

Key words: stars: luminosity function, mass function — open clusters and associations: individual: Bky 86

\section{Introduction}

This is the second paper in a series on observations of OB clusters with the goal to derive the initial mass function (IMF) of their low mass stars. Knowledge of the IMF in selected populations of stars is of interest in the derivation of the physical conditions leading to the formation of these stars. These conditions might be significantly different if a star forming event is slanted towards the formation of high or of low mass stars. Several previous reports, such as of NGC 3293 by Herbst \& Miller (1983) - for a review see Scalo (1986) - suggested a possible turnover in the IMF for stars of masses less than about $3 m_{\odot}$. However, this reported turnover occurred at the lower end of the observed mass range, and may be due to selection effects (Scalo 1986). Herbst \& Miller's result was based on the analysis of photographic plates very close to the detection limit.

A previous paper (Ninkov et al. 1995) was concerned with the central region of IC 1805 in the OB association Cyg OB2. In that cluster, many stars are thought to be pre-MS stars. No turnover in the IMF was found

Send offprint requests to: H.J. Deeg at the Instituto de Astrofisica de Canarias

${ }^{\star}$ Table 1 only available in electronic form at the CDS via ftp 130.79.128.5 for masses as low as $2.5 m_{\odot}$, and the IMF has a slope of $\Gamma=-1.38 \pm 0.19$ in the mass range of $2.5-30 m_{\odot}$. This slope is similar to that measured by other investigations of $\mathrm{OB}$ clusters with current starforming activity, such as NGC 6611 by Hillenbrand et al. (1993).

In this paper, photometric results based on a CCD survey of the core of the young open cluster Berkeley 86 (furthermore Bky 86) are presented. The core of this cluster is located at $l=76.7^{\circ}, b=1.3^{\circ}$. Blaha \& Humphreys (1989), and Leizawitz (1988) proposed Bky 86 as one of three nuclei for the OB association Cyg OB 1, the others being NGC 6913 (Walker \& Hodge 1968) and IC 4996 (Hoag \& Applequist 1965). However, Garmany \& Stenzel (1992) question the role of Bky 86 and the other two subclusters within Cyg OB 1, as the number of O stars in these clusters is relatively small compared to the total number of $\mathrm{O}$ stars that define Cyg OB 1. Furthermore, the earliest star in Bky 86 is only of type O9, making the relationship between Bky 86 and Cyg OB1 uncertain. The most extensive work on Bky 86 to date is by Forbes (1981) and Forbes et al. (1992, henceforth F92). Their $U B V$ photometry of 40 of the regions' brightest stars confirmed about half of them as cluster members, and they measured the spectral types of the brightest 9 stars. They derive a distance modulus of $11.01 \pm 0.20$, implying a distance of $1590 \pm 150$ pc, and a reddening of $0.8 \lesssim E(B-V) \lesssim 1.2$. From the 
evolutionary stage of the brightest member stars of Bky 86 , they deduce an age for Bky 86 of $5 \pm 1$ Myrs, with no evidence for current star forming activity. They suggest that Bky 86 is a member of the Cyg OB 1 association. The variable extinction across Bky 86 is a consequence of a foreground dust cloud at a distance of $900 \mathrm{pc}$, which is centered around far infrared source No. 4 of Campbell et al. (1982) and includes the nearby cluster Berkeley 87 .

After the following section on the observations, Sect. 3 covers the CCD image reduction, the photometry, and the procedure to obtain intrinsic stellar colors and magnitudes. In Sect. 4, the photometric data are used to extract probable member stars of Bky 86 from the sample. Bky 86 's IMF is derived and discussed in Sect. 5, and Sect. 6 gives a summary.

\section{Observations}

In the summer of 1993, a program of CCD observations of several galactic OB clusters, chosen from the list of Garmany \& Stenzel (1992) was carried out. Among these observations, Bky 86 was chosen for further analysis based on the quality of the data obtained. CCD images of Bky 86 were taken on the 5-6th of August, 1993, at the 0.9 m Monterey Institute for Research in Astronomy (MIRA) Telescope at the Oliver Observing Station in California. The CCD camera was equipped with a Kodak KAF 4200 chip with $2044 \times 2032$ pixels. The characterization of that chip is described by Deeg \& Ninkov (1995). The image scale at that telescope was $0.202 \mathrm{arcsec} / \mathrm{pixel}$. The useful field of view had a 6.2 arcmin side length, corresponding to an area of $8.23 \mathrm{pc}^{2}$ at the cluster distance.

The cluster was imaged through $U, B, V, R$ and $I$ filters in the Johnson-Cousins $U B V R I$ system, following the prescriptions of Bessell (1990). For each filter, exposures ranging from several seconds to several hundred seconds were taken, with total exposure times of $1270 \mathrm{~s}$ for the $R$ and $I$, and about 2100 s for the $U, B$, and $V$ filters. The staggering of exposure times was necessary to circumvent the limited dynamical range inherent to the camera's 12 bit analog-to-digital converter. Typical FWHMs of the stellar point spread function ranged from 1.5 to 1.8 arcsec. Sky flat fields, dark frames and bias frames were taken in the usual manner.

\section{Data processing}

The image processing of the raw data was performed using IRAF. The chip displayed a significant, and spatially varying deviation from linearity of about $25 \%$. This condition became apparent when cross-calibration between images taken with different exposure times was attempted. As a consequence of this, laboratory exposures were performed leading to a detailed characterization of the CCD, and to the derivation of a correction function (Deeg \& Ninkov 1995). After correction, there remained a linearity error not exceeding $5 \%$. This error of $\delta_{\text {lin }}=0.05$ mag was assigned to all measured magnitudes. For stars closer than about 100 pixels to the frame border, $\delta_{\text {lin }}$ was larger than 0.05 mag. Consequently, these stars were omitted in the reduction, and the useful field of view was limited to a size of $(6.2 \mathrm{arcmin})^{2}$.

The DAOPHOT package was used to find the positions for the stars on the images, and an initial sample of 799 stars was obtained, with a limiting $V$ magnitude of $\approx 22.5$ mag. Bright stars were measured on the frames with short exposure times, as they were oversaturated in the longer exposed frames. On uncrowded frames from short exposures, aperture photometry was performed with the IRAF-'phot' task, using an aperture of 3.5 times the FWHM of the frame's typical psf. Aperture photometry was found to produce more consistent results than psf fitting photometry, which was only used (with the daophot'allstar' task) for frames with the longest exposure times, where crowding became significant. Typically between $20-$ 50 stars of intermediate brightness could be measured on several frames of different exposure times, allowing crosscalibration of the instrumental magnitudes of the faintest stars against instrumental magnitudes of the bright stars. A cross-calibration error $\delta_{\mathrm{cc}}$ of about $0.017 \mathrm{mag}$ is introduced in each cross calibration step, leading to $\delta_{\mathrm{cc}} \approx 0.05$ mag for the photometry of the faintest stars (with three cross-calibration steps).

Errors from photon count statistics, $\delta_{\mathrm{pc}}$, were determined for each measured star. From our initial sample of 799 stars, faint stars where rejected, if $\delta_{\mathrm{pc}}>0.15 \mathrm{mag}$ in any one of the bands of $V, R$ or $I$. Additionally rejected was a small number of unresolved double stars, and stars within the artifacts produced by nearby saturated bright stars. Reliable $V, R$ and $I$ magnitudes could be measured for 544 stars. The field of Bky 86 with these stars is shown in Fig. 1. Photometry in $B$-band could be obtained on a subset of 196 stars, and in $U$-band, photometry was possible for 60 stars only.

The instrumental magnitudes were zero-pointed against the F92 data, after applying color correction terms. The color correction terms where found from photometry of 12 standard stars in NGC 7790 (Christian et al. 1985), taken with the same CCD-filter setup at the University of Rochester's Mees observatory on the 12th December 1993. The color correction terms were determined with the "Photom" software (Beckert 1991) and are:

$$
\begin{gathered}
\Delta v_{\mathrm{ccd}}=\Delta V-(0.081 \pm 0.04)(V-R) \\
\Delta(u-b)_{\mathrm{ccd}}=(1.0 \pm 0.1) \Delta(U-B) \\
\Delta(b-v)_{\mathrm{ccd}}=(0.87 \pm 0.08) \Delta(B-V) \\
\Delta(v-r)_{\mathrm{ccd}}=(0.83 \pm 0.04) \Delta(V-R) \\
\Delta(r-i)=(1.23 \pm 0.04) \Delta(R-I)
\end{gathered}
$$




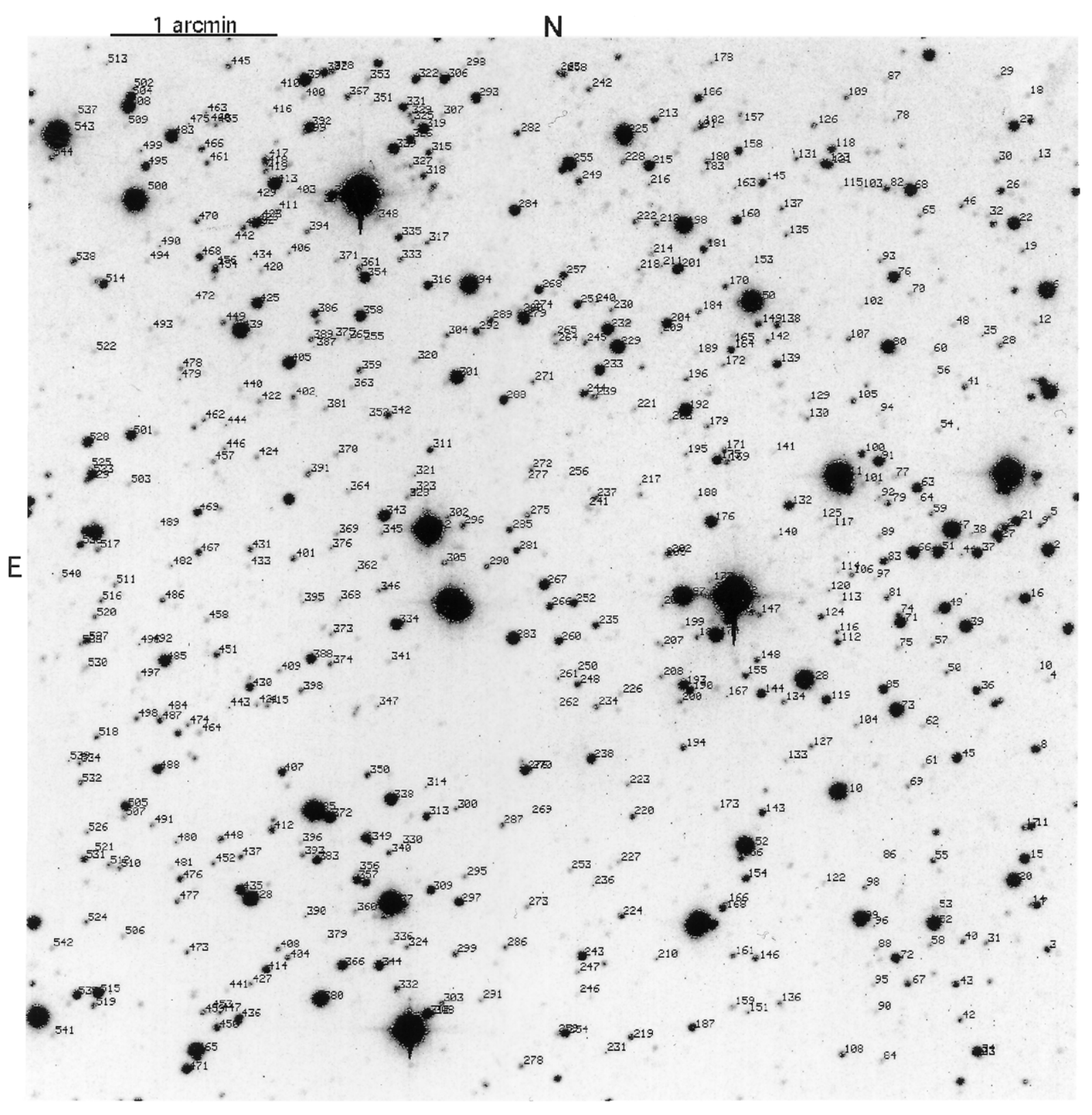

Fig. 1. $I$ band CCD frame of Berkeley 86. Where crowding makes the identifications of star numbers difficult, we refer to the stars' positions in Table 1

A reliable determination of the zero-points was not possible from these observations, and we zeropointed our photometry to the $U B V$ magnitudes of the 23 stars given by F92 (their numbers 1-11, 22-33, and 39), which were common with our field of view. No star in our frames had any prior determination of its $R$ or $I$ magnitude. The $V-R$ and $R-I$ colors were therefore calibrated based on the expected $V-R$ and $R-I$ colors of the 6 stars for which spectral classes (based on spectroscopy) are available from F92. These stars' intrinsic colors were determined using the color vs. spectral class relation from Johnson (1966), converted to the Cousins VRI system (Bessell 1979). The intrinsic colors were then corrected for extinction. The extinctions $E(B-V)$ for these stars were taken from F92's values and were converted to $E(V-I)$ using the relationship given by Dean et al. (1978). Using this $E(V-I)$ value, the extinctions $E(V-R) \approx 0.45 E(V-I)$, and $E(R-I) \approx 0.55 E(V-I)$ were derived by interpolating the interstellar extinction curve of Savage \& Mathis (1979) for the center wavelengths of the Cousins $R$ and $I$ band. The internal uncertainties in our results, when comparing with stars also measured by F92, are approximately 0.05 
mag for all colors. This error for the magnitude calibration, $\delta_{\mathrm{mc}}$, was assigned to all stars.

The accumulated photometric errors for each star, $\delta_{\text {tot }}$ $=\left(\delta_{\mathrm{lin}}^{2}+\delta_{\mathrm{cc}}^{2}+\delta_{\mathrm{pc}}^{2}+\delta_{\mathrm{mc}}^{2}\right)^{1 / 2}$, are plotted versus magnitude in Fig. 2 for the $V$ band. For the brightest stars, errors due to the nonlinearity of the CCD camera, $\delta_{\text {lin }}$, and errors due to the absolute magnitude calibration, $\delta_{\mathrm{mc}}$, dominate. Towards fainter magnitudes, the uncertainties increase in two distinct steps at $V \approx 12$ and $V \approx 15$. These are errors from the additional cross-calibration steps $\left(\delta_{\mathrm{cc}}\right)$ needed for fainter stars. For the faintest stars, uncertainties from photon count statistics, $\delta_{\mathrm{pc}}$, dominate. In the $R$ and $I$ bands, the photometric errors were lower, as the sensitivity of the CCD camera in these bands was higher.

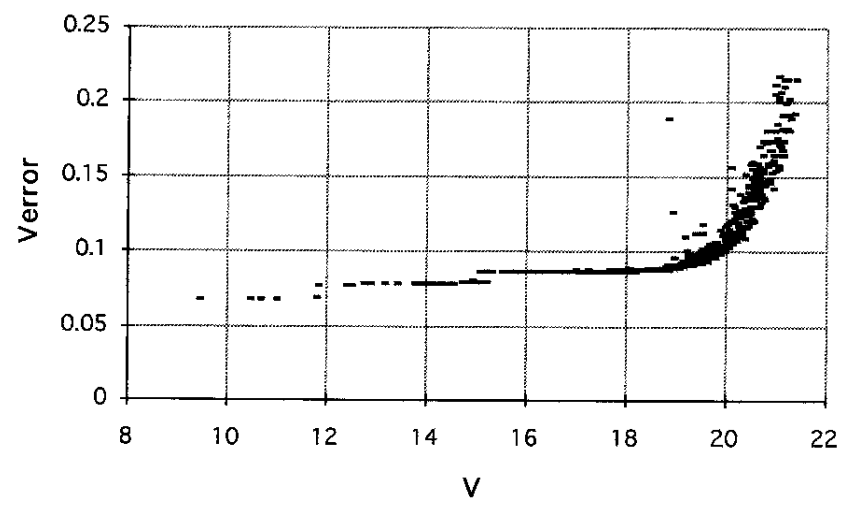

Fig. 2. Accumulated errors in $V$ band photometry versus the observed $V$ band magnitude for the 544 stars of the sample

The photometric results and epoch J2000 positions for the 544 stars with measured $V, R$, and $I$ magnitudes are given in Table 1, which is available electronically at CDS. The star positions are precise to about $1^{\prime \prime}$ and are intended for identification purposes only. They are based on the positions of some stars which are listed in the Hubble Guide Star Catalog. Table 1 also includes masses for those stars which are probable members of Bky 86 (selected from case (i), see later in text).

To obtain the stars' intrinsic colors in $V, R$ and $I$, extinction corrections have to be applied to the observed magnitudes. The extinction in Bky 86 varies and F92 provide a map of the approximate spatial variation of the $E(B-V)$ values. Across the field of view of our CCD, $E(B-V)$ varies between 0.89 and 1.25 mag. An approximate value of $E(B-V)$ was derived for each star by interpolation between contour lines in the F92 map, and is included in Table 1. Due to the absence of known $B-V$ colors for most stars, the earlier mentioned conversion of $E(B-V)$ to $E(V-I)$ by Dean et al. could not be used. Simplified conversions based on an interpolation of Savage and Mathis' extinction curve were employed instead, with: $E(V-R)=0.56 E(B-V)$ and $E(R-I)=0.68 E(B-V)$. $V$ band magnitudes were dereddened using the standard relationship $A_{v}=3.10 E(B-V)$ and converted to abso- lute magnitudes, $M_{v}$, using a distance modulus to Bky 86 of 11.01. It should be noted, that $A_{v}$ is of the order of 3 magnitudes.

\section{Interpretation of the data}

\subsection{Completeness of the sample}

The results derived in this paper are based only on the $V R I$ photometry, since $B$ and $U$ band photometry could be obtained for a subsample only. The initial sample with 799 stars extended to stars about 1 magnitude fainter than the limit for which reliable photometry could be obtained, which is at about $V=21.5$. Based on the star counts (Fig. 3 ), and on the shape of the mass distribution functions later discussed in this paper, we are confident that for $V R I$ photometry, our sample is complete to $V \lesssim 19.5$, with the exception of a few close double stars and some faint stars which are very close to bright ones. At the distance and typical extinction of Bky $86, V=19.5$ corresponds to stars of $M_{v} \approx 5.3$, which are G7V stars with masses of $\approx 0.9 \mathrm{~m}_{\odot}$.

Reliable photometry could not be obtained for four of the brightest stars within our field of view, and these stars were omitted from the sample. F92 classified three of them as members, based on spectroscopic data (F92's Nos. 3, 4, and 9 , the non member is No. 8). These three member stars were included in the cluster mass functions, using the magnitudes by F92.

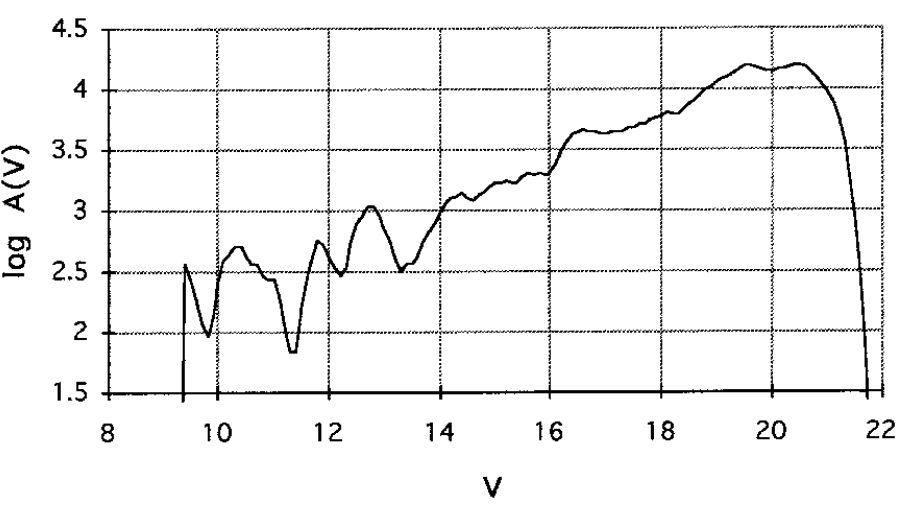

Fig. 3. Star numbers in Bky 86 as a function of apparent magnitudes. A is in units of the number of stars per $V$ magnitude and per square degree. $\log A$ is about 0.5 lower than the average values for $b=0^{\circ}$ in Allen (1976), owing mostly to the strong extinction in the direction of Bky 86

\subsection{Extraction of the member stars of Bky 86}

For the determination of the correct IMF of a cluster, a separation of the stars into cluster-member and field stars is necessary. Two methods are employed below: estimates for the numbers of field stars, and a photometry-based extraction of cluster member stars which depends on their 
proximity to the cluster-MS in an HR diagram. It should be noted, that secure membership identifications can only be gained by determination of the stars' velocity or spectral type.

Field star counts were performed by F92 around Bky 86. From star counts to a limiting magnitude of $\approx 20 \mathrm{mag}$ on Palomar E plate within $13^{\prime}$ around the cluster center, they derive a field star density of about 2.72 stars per arcminute ${ }^{2}$. This density implies about 105 field stars with $V<20$ in our f.o.v.. For bright stars with $V<14.7$, a lower limit is given by the 8 stars known as non-members from F92. For further estimates of the field star counts in Bky 86, we assume (from these two values) that the number of field stars brighter than $V$ within our f.o.v. is given by: $\log N_{\text {field }}(V)=-8.82+8.33 \log V$. This function was chosen, because the tabulations of field star distributions by Allen (1976) or Sears \& Joyner (1928) can be fitted excellently by functions of this kind. For $V \leq 18.5$, as used below, 55 field stars are expected.

For the interpretation of $U B V$ photometry of clusters with variable extinction, it is common to plot the stars' position in a $(U-B)$ versus $(B-V)$ diagram. This is done for the 60 stars with $U B V R I$ photometry in Fig. 4 . The intersection of a star's dereddening path (parallel to the arrow in Fig. 4) with the intrinsic MS is interpreted as a star's position on the MS. If the length of the reddening path is within constraints set by the typical cluster extinction, the star is judged to be a cluster member. Examples of this method can be found in F92, Massey et al. (1989), and Massey \& Thompson (1991). For $U B V$ photometry this method is feasible, since the reddening path points away from the MS in color-color diagrams. For $V R I$ photometry, the reddening path in any combination of $V, R$ and $I$ colors is much closer aligned with the MS. Still, the $(V-R)$ versus $(R-I)$ color-color diagram (Fig. 5) shows that most stars are shifted in color to the reddened cluster MS. Figure 5 separates between stars brighter and fainter than $V=18.5$. Whereas the brighter stars are well aligned with the reddened MS, the fainter ones show considerable scatter. A comparable situation is evident in a $V$ versus $V-R$ diagram (Fig. 6). Bright stars are generally close to the cluster-MS. In this diagram, MS's at several foreground and background distances are included; uniform extinction per distance was assumed. The bulk of the fainter stars, with $V>18.5$, is in a region where the cluster MS and foreground MS's cross, and the background MS's get very close to the cluster MS. The membership assessment gets continuously more unreliable for fainter stars due to the increasing fraction of field stars, increasing photometric errors and the ambiguities in the placements on any particular MS. We therefore limited membership determination to stars with $V<18.5$.

The stars' intrinsic colors and their position relative to the cluster MS is shown in Fig. 7. To the right of the cluster MS, appreciable numbers of stars are found only

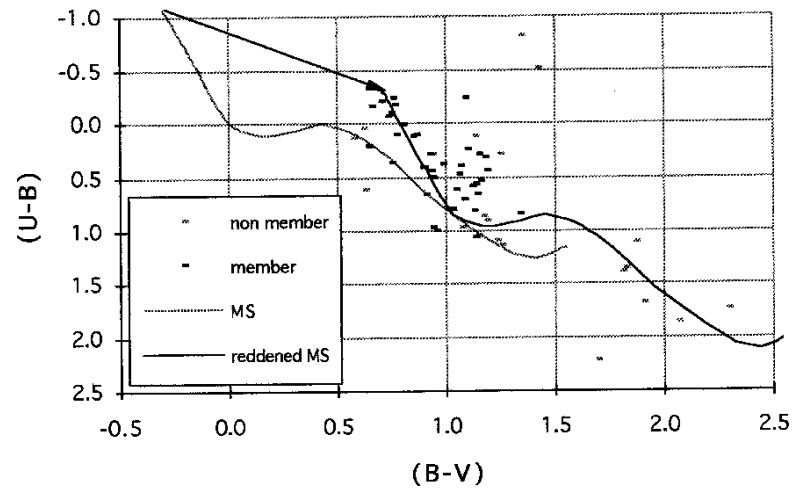

Fig. 4. $U-B$ versus $B-V$ color diagram for the 60 stars with $U B V$ photometry. The arrow indicates the average extinction of Bky 86 of $E(B-V)=1.02$, and $E(U-B)=0.85$, which shifts the intrinsic MS (dotted line) to the MS at the cluster's extinction (solid line). Stars that are selected as cluster members based on VRI photometry (from case (i), see Sect. 4.2) are indicated in black, the others in gray. The spread of the member stars around the intrinsic MS is a result of photometric errors (which are larger in $U-B$ and $B-V$ as for $V R I$ colors, and are as big as $0.25 \mathrm{mag}$ for the faintest stars with quoted $U-B$ colors, see Table 1 ) and of varying extinction within the cluster, which ranges between 0.9 and 1.25 for $E(B-V)$

for $M_{v}>-0.5$, corresponding to MS masses of less than $4.5 m_{\odot}$. If these stars would be evolved cluster members, they would need to be older than $10^{8}$ years, which is incompatible with estimated cluster ages of $5 \pm 1$ Myrs (F92) or 41 Myrs (Leizawitz 1988; Lynga 1987). Furthermore, stars of that age would be expected to have dispersed away from the cluster of their origin. It is however feasible that some of these stars are pre-MS stars, as newer studies about the pre-MS stellar evolution (D'Antona \& Mazzitelli 1994; Bernasconi \& Maeder 1996) indicate preMS timescales on the order of $10^{6-7}$ yrs. A large fraction of the stars to the right of the MS can however be expected to be background giant stars. Also not excluded can be, that some cluster members suffer very high extinction. Among stars located within $\Delta M_{V} \approx 0.75$ above the cluster MS, there may be a significant fraction of cluster members that are binaries. The possible effects from preMS stars and from binaries onto the derived mass function are discussed later. The few stars to the left of the MS are likely foreground stars.

For an initial identification of member stars, a strip along the MS on the $M_{v}$ vs. $(R-I)$ diagram (Fig. 7) was defined, inside which stars are considered to be members. The major uncertainty of this method is the choice of the width of the strip along the MS. Two choices with this criterion were used:

(i) stars are considered members if they are not farther from the MS then $0.3 \mathrm{mag}$ in $M_{v}$ and $0.1 \mathrm{mag}$ in $R-$ 


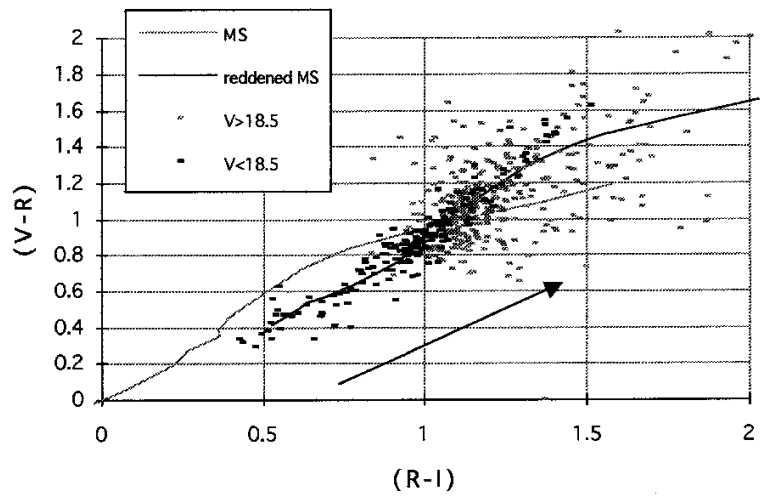

Fig. 5. Two-color diagram of the observed colors of Bky 86. The arrow indicates the cluster reddening path, based on an average value for Bky 86 of $E(B-V)=1.02 \mathrm{mag}$. The MS curves are based on Bessell (1990) for Cousins VRI filter specifications. MS colors form Johnson (1966) converted to Cousin's $V R I$ colors were used for stars earlier than B3. The gray line is the MS at the star intrinsinc colors, the black line is the MS reddened with the cluster reddening path. All colors are in the Cousins system

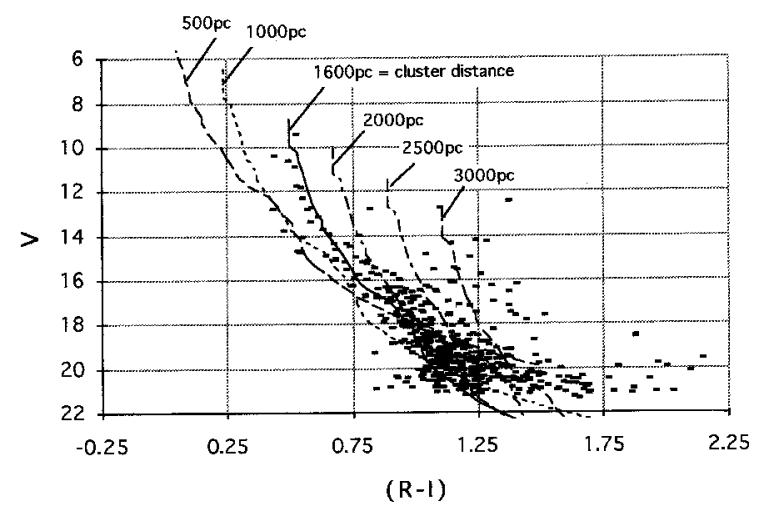

Fig. 6. $V$ magnitude versus $R-I$ colors of the sample. Overplotted are luminosity class $\mathrm{V}$ mainsequences at the distances indicated. The mainsequence at $1600 \mathrm{pc}$ (solid line) is at the approximate distance of Bky 86 and assumes an extinction of $A_{v}=3.16$ and a reddening of $E(R-I)=0.69$. The other mainsequences were calculated assuming constant extinction per distance. Stars in the lower right are most likely background giant stars

\section{These limits represent the typical uncertainties of the} photometry.

(ii) as above, but using $0.15 \mathrm{mag}$ in $M_{v}$ and $0.05 \mathrm{mag}$ in $R-I$.

Results are given in Table 2. In case (i), out of a total of 180 stars with $V<18.5$, there were 54 non-member stars (Fig. 7), which is in good agreement with the field star counts mentioned before. Figure 8 is an enlargement of Fig. 7, which shows all stars common to F92 and this work. The membership status of these stars, as determined by F92, as well as by case(i), is indicated. Of the 20 stars

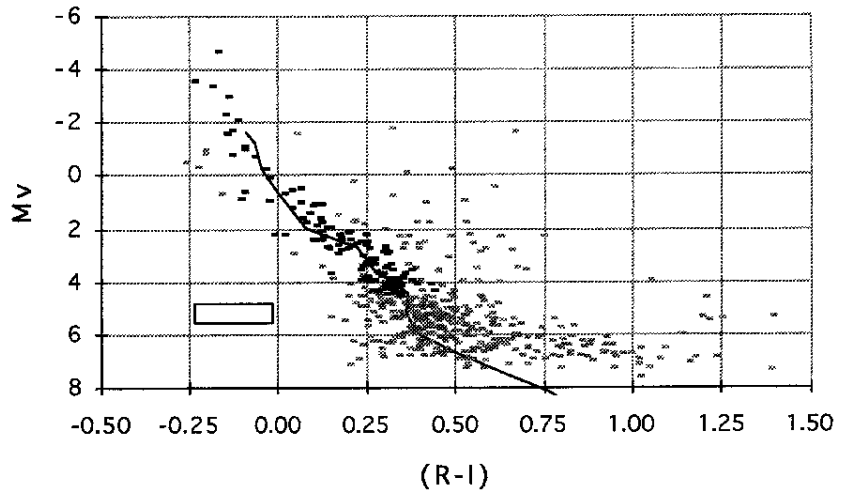

Fig. 7. As for Fig. 5, but the data were corrected for reddening and a distance modulus of 11.01 was applied. The extinction was calculated for each star individually, based on its position in F92's map of Bky 86's extinction. The stars that were selected to be members of Bky 86 in case(i) are marked in black, the remainder are in gray. The rectangle in the lower right shows the size of the box for case(i) that defines the selection strip for membership along the MS. The MS based on Bessell (1990) (solid line) was used, except for the brightest stars $\left(m_{v}<-1.59\right)$, where the Johnson (1966) MS was used

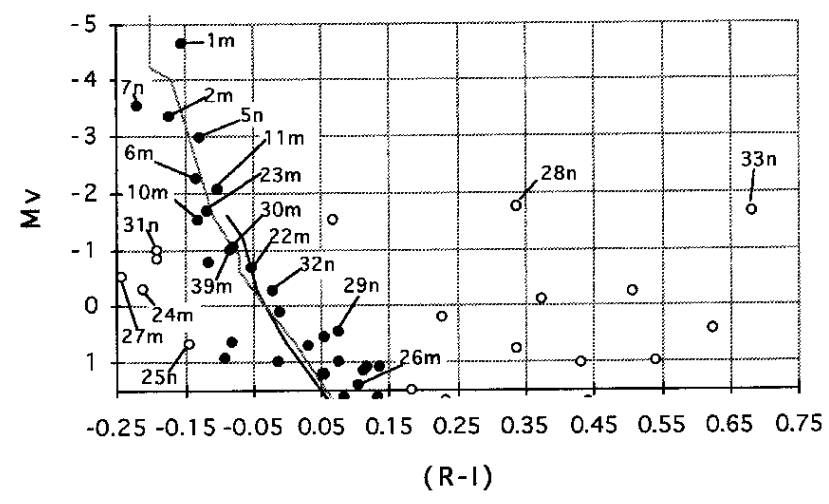

Fig. 8. An enlargement of Fig. 7 for the stars common to F92 and to this work, giving a comparison between the membership assessments in these works. The numbers are the identifications used by F92, 'm' or ' $n$ ' indicates their assessment for member or nonmembership. Solid circles are members selected from case(i) in this work, hollow circles are nonmenbers. The gray line is the MS based on Johnson (1966), the black line is the MS from Bessell (1990)

in common, 14 stars as selected as members according to case(i), 12 stars are members according to F92, and 10 stars are selected as members by F92 and by case (i). The more stringent selection criteria of case (ii) gave a much poorer agreement here.

\section{The initial mass function}

The goal in measuring the mass distribution of this ensemble of stars is the derivation of the initial mass 
Table 2. Results for IMFs with varying selection criteria

\begin{tabular}{cccc}
\hline $\begin{array}{c}\text { selection criteria } \\
\text { for membership (see texl) }\end{array}$ & $\mathrm{N}^{1}$ & $\Gamma$ & $\begin{array}{c}\text { mass range } \\
{[\mathrm{m} / \mathrm{m} \text { o] }}\end{array}$ \\
\hline (i) Mv: $0.3 \mathrm{mag}, \mathrm{R}-\mathrm{I}: 0.1 \mathrm{mag}$ & 126 & $-1.3 \pm 0.3$ & $1.2-20$ \\
(ii) Mv: $0.15 \mathrm{mag}, \mathrm{R}-\mathrm{I}: 0.05 \mathrm{mag}$ & 90 & $-1.4 \pm 0.4$ & $1.2-20$ \\
subset of (i) for $\log \mathrm{m}<0.55$ & 110 & $-2.7 \pm 0.5$ & $1.2-3.5$ \\
subset of (i) for $\log \mathrm{m}>0.55$ & 16 & $\geq-0.9$ & $3.5-20$ \\
All stars selected with $\mathrm{V}<18.5$ & 180 & $-1.4 \pm 0.3$ & $1.2-20$ \\
\hline
\end{tabular}

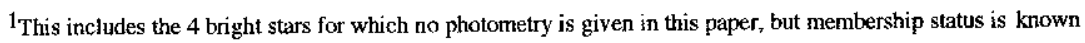

from $\mathrm{F92}$.

function (IMF), which describes the mass distribution of the stars at their formation. In the following discussion we use the generally accepted notation, as it is employed in the thorough review by Scalo (1986). The direct result of the photometry, after rejecting non-members and nonMS stars, is the present luminosity function of Bky 86, $\phi_{\mathrm{ms}}\left(M_{v}\right) \mathrm{d} M_{v}$, which describes the number of MS stars with absolute magnitudes between $M_{v}$ and $M_{v}+\mathrm{d} M_{v}$. $\phi\left(M_{v}\right)$ can be converted into a mass spectrum, which is called the present day mass function (PDMF), $\phi_{\mathrm{ms}}(\log m)$, with the use of a mass-luminosity relation. For this conversation we used the $M_{V}-\log m$ relationship given in Table IV of Scalo's review.

An IMF normalized per unit area is usually denoted by $\xi(\log m)$, which gives the number of stars with mass $(\log m)$, born per $\log (m)$ mass interval and per $\mathrm{pc}^{2}$. To characterize the shapes of IMFs, the steepness of an IMF within some mass range is a useful parameter. It is given by:

$$
\Gamma=\frac{\mathrm{d} \xi(\log m)}{\mathrm{d}(\log m)}
$$

If all of the stars born in the cluster, and with masses $m<m_{0}$ are still on the MS, then the IMF is identical to the PDMF for $m<m_{0}$. A case for the presence of most member stars of Bky 86 being on the MS has already been made in the section dealing with the selection of member stars - i.e. no star formation is occuring now, and all stars have evolved onto the MS. The only exception are a few very bright stars with $M_{v}<-2.5$, that have already evolved slightly off the MS (F92). These bright stars have masses larger than $10 M_{\odot}$, leading us to conclude that the IMF is identical to the PDMF for $m<10 M_{\odot}$.

IMF's were derived this way from stars selected using the cases (i) and (ii) introduced previously. The slopes and applicable mass ranges of the derived IMFs are included in Table 2. For stars selected with case(i), the masses of the individual member stars are included in Table 1. The IMF for case(i) is given in Table 3, and Fig. 9 is a plot of the IMF as a continuous distribution. The distribution was created by binning of the sample into a large number of narrow bins of $0.01(\log m)$, smoothing with a triangular kernel with a base-width of $0.09(\log m)$ for $(\log m)$ $<0.5$ (a width of 0.27 for the higher mass bins was used to account for the small numbers of stars), and application of appropriate normalization constants. The distribution function shows two dips at $(\log m) \approx 0.15$ and at $(\log m)$ $\approx 0.32$, which is also present in distribution functions derived from case(ii). However, we abstain from weighting these dips with astrophysical significance, unless similar features are reported in comparable clusters.

Unresolved binaries would lie above the single-star MS in the $M_{V}$ vs. $R-I$ diagram (Fig. 7 ). The MS for binaries of equal masses is $0.75 \mathrm{mag}$ above the normal MS; binaries with more unequal mass ratios are closer to the single-star MS. This means, that a certain fraction of stars above the extraction strips of cases (i) or (ii) may actually be members. As the extraction strips, particularly in case (i), already have a considerable width, we expect that most binaries are already included as cluster members in these cases. Mermilliod \& Mayor (1989) found a binary percentage of $25-33 \%$ in five open clusters, but the percentage of binaries among the supposed non-cluster-members above the MS is difficult to estimate. To test a case where the influence of binaries onto the mass functions would be extreme, we choose case (ii), which is a relatively narrow strip along the MS and should not contain many binaries. A second parallel strip, shifted 0.75 mag above the normal MS was used to select additional stars that are possible binaries. If $50 \%$ of these additional stars (i.e. assuming that half of these stars are binaries) are included into the derived IMF, only marginal changes in the lowest mass bins are found. An investigation of binaries in the cluster King 2 by Aparicio et al. (1990) also found only minor effects from their inclusion into the IMF, whereas Sagar \& Richter (1991) derive that binary-free IMF's with $\Gamma=-1.5$ would flatten by about $\Delta \Gamma=0.17$ from the assumption of $30 \%$ binaries with random stellar mass distributions. 


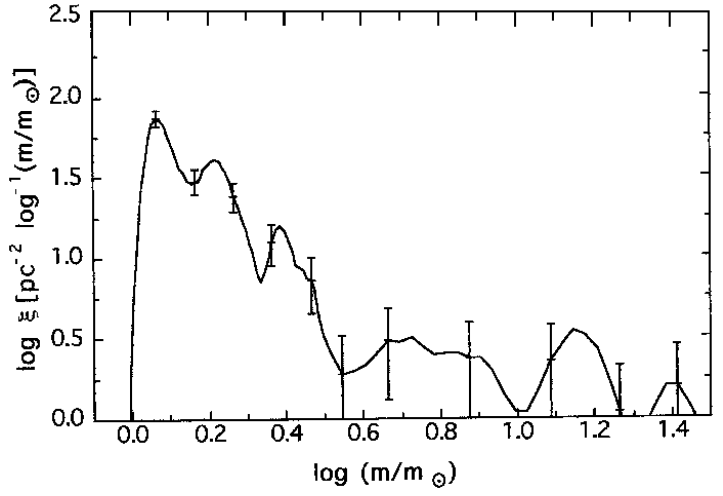

Fig. 9. Initial mass function of Bky 86 for case(i). The IMF is normalized to a unit area of $1 \mathrm{pc}^{2}$. Error bars are based on the number of stars in those bins that contribute to the smoothing kernel

Table 3. The IMF of Bky 86 for case(i)

\begin{tabular}{cccc}
\hline $\begin{array}{c}\text { Mass range } \\
\text { [m/mo] }\end{array}$ & $\begin{array}{c}\log \left(\mathrm{m} / \mathrm{m}_{\odot}\right) \\
\text { ccntcr of bin }\end{array}$ & $\mathrm{N}$ & $\left.\begin{array}{c}\log \xi \\
{\left[\left(\log \mathrm{m} / \mathrm{m}_{\odot}\right)^{-1}\right.}\end{array} \mathrm{pc}^{-2}\right]$ \\
\hline $1.1-1.4$ & 0.10 & 56 & 1.85 \\
$1.4-1.8$ & 0.20 & 30 & 1.60 \\
$1.8-2.2$ & 0.30 & 11 & 1.13 \\
$2.2-2.8$ & 0.40 & 10 & 1.09 \\
$2.8-3.5$ & 0.50 & 3 & 0.56 \\
$3.5-7$ & 0.70 & 7 & 0.45 \\
$7-14$ & 1.00 & 6 & 0.39 \\
$14-20$ & 1.30 & 3 & 0.09
\end{tabular}

To evaluate the possible influence of pre-MS stars onto the mass function, we converted the photometric $V$ and $I$ magnitudes to luminosities and effective temperatures, as described by Ninkov et al. (1995). An overlay of the preMS tracks from Bernasconi (1996) with the Bky 86 data (Fig. 10) shows that only relatively few stars which are not selected as members from case(i) are in the pre-MS region on the HR diagram. But even in the extreme case, that all of these few stars are pre-MS stars, their influence onto the derived IMF is negligible. We also note, that indicators for the current production of young stellar objects in Bky 86, such as a strong $\mathrm{H} \alpha$ emission, are absent.

For further discussion, the IMF derived from case (i) without the inclusion of binaries or pre-MS stars is adopted. This IMF has a steepness of $\Gamma \approx-1.3 \pm 0.3$, taken over the entire mass range of $1.2-20 m_{\odot}$. The IMF is relatively steep in the mass range $1.2 m_{\odot}-3.5 m_{\odot}(0.08 \lesssim$ $\log m \lesssim 0.5)$, with $\Gamma \approx-2.8 \pm 0.5$. This is however most likely the result of an underabundance of stars in the mass range of $0.5<\log m<1$. While this 'hole' may be the result of a mass separation in the cluster core, it may also be simply a consequence of the relative small number of higher mass stars available. The addition of 5-8 stars in the 'hole' would make it disappear. The apparent flatness of the IMF with $\Gamma \gtrsim-0.9$ at masses of $m>3.5 m_{\odot}$ may also be purely a result of the small sample size, as there are only 16 stars in that mass range. The IMF generated from case (ii) is slightly steeper (Table 2), but otherwise shows the same details as the adopted IMF.

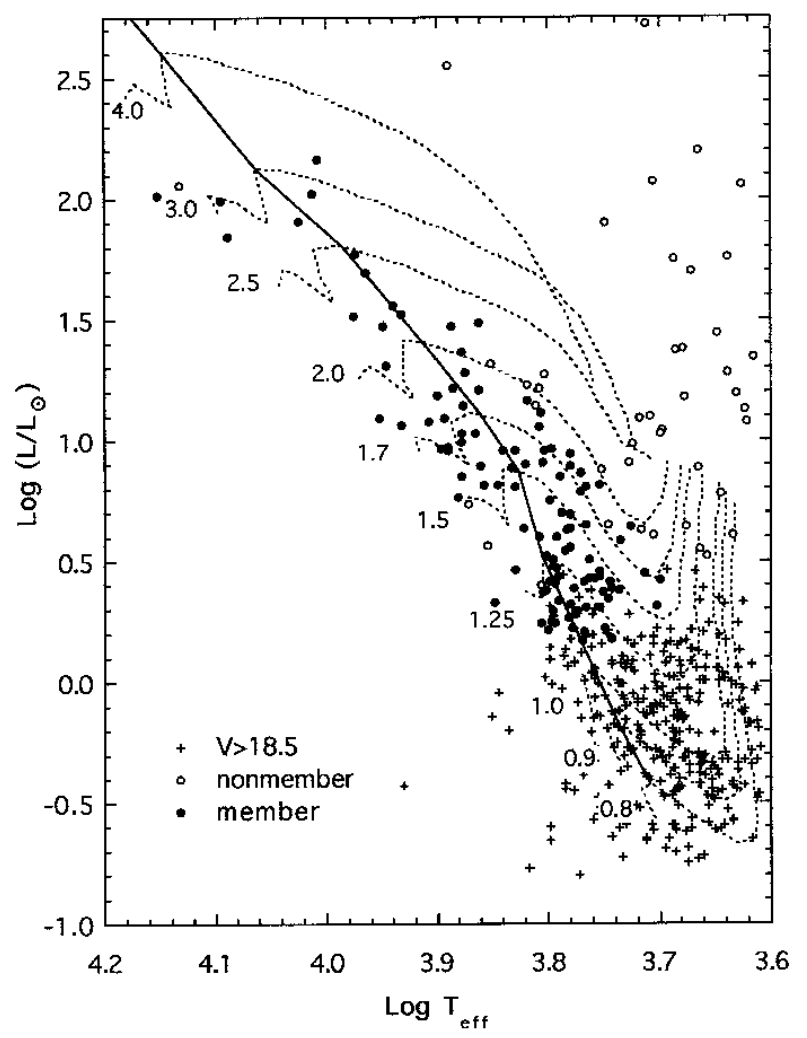

Fig. 10. Pre-MS tracks for $0.8-4 M_{\odot}$ (dotted lines, from Bernasconi 1996), overlaid with data from the Bky 86 field. The solid line indicates the ZAMS. Solid circles are Bky 86 members, from case(i). Only relatively few of the stars which are selected as non-members (open circles) are in the region of the pre-MS tracks. If all of them (a total of 16) are considered cluster members and they are assigned the masses of their pre-MS track, their impact onto the IMF is still negligible. Crosses are stars with $V>18.5 \mathrm{mag}$, for which no membership determination was performed

Most of the recent studies of young clusters have derived IMFs that are comparable to the IMF found here for Bky 86. The study of Phelps \& Janes (1993) of 8 young open clusters gave an average of $\Gamma=-1.4 \pm 0.13$ in the mass range $1.4-7.9 \mathrm{~m}_{\odot}$. Similarly, the study of IC 1805 by Ninkov et al. (1995), and the compilation of results from other clusters therein, indicates an average IMF steepness of $\Gamma=-1.38 \pm 0.19$. The result from Bky 86 is another indication, that the IMF of most clusters is consistent with a Salpeter IMF, with $\Gamma=-1.35$. There are 
few exceptions known: NGC 581 has a slope of $\Gamma=-1.78$ (Phelps \& Janes 1993) and the IMF derived for the cluster NGC 129 by Frolov (1975) is very steep with $\Gamma \approx-2.8$, but it was determined only in the narrow mass range of 2 to $3.5 m_{\odot}$. The mass range over which IMFs are determined appears to be crucial for the interpretation of the steepness of the average IMF. A steeper IMF is derived for Bky 86 , if the fitting is made to a lower upper mass limit. A weak dependence of this kind has also been demonstrated by Scalo 1986 (his Fig. 34). In an extreme case, fitting a powerlaw IMF to Bky 86 in the mass range 1.1-3.5 $m_{\odot}$ gives $\Gamma=-2.7$, comparable to the results on NGC 129 . These effects are most likely the results of gaps, or dips, in the IMF, as is apparent in Bky 86 in the mass range of $3-10 m_{\odot}$. This may lead to the interpretation of narrow sections of an IMF as being unusual steep. Several young clusters with comparable age to Bky 86 appear to have similar gaps at the high mass end (Phelps et al. 1976). Even with the relatively small number of high mass stars involved, these gaps have low probabilities to be random occurrences, so physical causes cannot be excluded.

Our derived IMF for Bky 86 is in contrast to some studies of $\mathrm{OB}$ clusters where indications of low mass turnovers in the IMF have been reported. Only few studies have extended to masses below $1.5 \mathrm{~m}_{\odot}$, limiting the opportunities for comparison with this work. One such study is the determination of a combined IMF from 117 stars of $\alpha$ Per, NGC 2224, Centaurus and Scorpius regions (Claudius \& Grosbol 1980) by Scalo (1986). These data produce a flat IMF in the mass range $2.5 m_{\odot} \lesssim m \lesssim 6 m_{\odot}$. Another study is that of NGC 3293 based on photographic data by Herbst \& Miller (1983), showing an IMF turnover at $m \approx 3 m_{\odot}$, and a falloff with $\Gamma \approx+2$ for masses down to $m \approx 1.5 m_{\odot}$. Although incompleteness or effects from membership- selection can easily cause such turn-overs, the authors attribute this turnover to real physical causes. No low mass turnover is indicated by the composite IMF generated by Taff (1974) from the data of 62 clusters, with $\Gamma \approx-1.8$ in the mass range of 1 to $10 m_{\odot}$. Similarly, the IMF derived for the cluster NGC 129 by Frolov (1975) shows no turnover or signs for flattening.

Attempts have been made to continue the IMF of Bky 86 towards fainter stars in the range of $18.5<V<20$. This lead to a continuation of the steep mass function with $\Gamma \approx-2.8$ towards about $0.85 m_{\odot}$, with no indications for a turnover above the completeness limit. Estimates of the fraction of field stars in this range are however very unreliable.

\section{Summary}

1. CCD images of the cluster Bky 86 were obtained and $V R I$ photometry for 544 stars within a radius of about $3^{\prime}$ of the center was determined. The completeness limit of the $V R I$ photometry is about at $V=20 \mathrm{mag}$; with a small fraction of stars being omitted due to blending. For a limited sample of brighter stars, $B$ and $U$ photometry is also derived.

2. A strong concentration of stars appears along the cluster-MS in a Hertzsprung Russell diagram of extinction corrected $M_{v}$ versus $(V-I)$ values.

3. Most stars close to the MS may be considered cluster members; the young age of the cluster leads to the assumption, that the cluster mass function is similar to the IMF.

4. The derived IMF has a slope of $\Gamma \approx-1.3 \pm 0.3$ in the mass range of 1.1 to $20 m_{\odot}$ under the assumption of no binaries; with a binary fraction of $30 \%$ it may be about $\Delta \Gamma \approx 0.2$ flatter. In the narrow range of 1.1 to $3.5 m_{\odot}$ it may be steeper, with $\Gamma \approx-2.7$. The IMF is much flatter at masses $>3 m_{\odot}$ with $\Gamma \approx-1$, but this region suffers from small number statistics. The break in the steepness of the IMF is likely the result of a gap in the IMF at $3-10 m_{\odot}$.

5. There is no turnover in the cluster IMF at masses above $1.1 \mathrm{~m}_{\odot}$, and there are no signs for one, albeit with lower confidence, at masses down to $0.85 m_{\odot}$.

Acknowledgements. The CCD sensor development was supported by an NSF IUCRC and a New York State CAT grant to the Center for Electronic Imaging Systems and a FAR Theodore Dunham Jr. Grant.

\section{References}

Allen C.W., 1976, Astrophys. Quantities. London: Athalone Press

Aparicio A., Bertelli G., Chiosi C., Garcia-Pelayo J.M., 1990, A\&A 240, 262

Beckert D., 1991, Photom version July 1991, Master's Thesis, Univ. of New Mexico

Bessell M.S., 1990, PASP 102, 1181

Blaha C., Humphreys R. M., 1989, AJ 98, 1598

Bernasconi P.A., Maeder A., 1996, A\&A 307, 829

Campbell M.F., Hoffman W.F., Thronson Jr. H.A., Niles D., Nawfel R., Hawrylycz M., 1982, ApJ 261, 550

Christian C.A., Adams M., Barnes J.V., et al., 1985, PASP 97, 363

Claudius M., Grøsbol P.J., 1980, A\&A 87, 339

D'Antona F., Mazzitelli I., 1994, ApJS 90, 467

Dean J.F., Warren P.R., Cousins A.W.J., 1978, MNRAS 183, 569

Deeg H.J., Ninkov Z., 1995, Opt. Engin. 34, 43

Forbes D., 1981, PASP 93, 441

Forbes D., English E., De Roberts M.M., Dawson P.C., 1992, AJ 103, 916 (F92)

Frolov V.N., 1975, Soviet Astrophys. J. Lett. 1, 156

Garmany C.D., Stenzel R.E., 1992, A\&AS 94, 211

Herbst W., Miller D.P., 1982, AJ 87, 1478

Hillenbrand L.A., Strom S.E., Vrba F.J., Keene J., 1992, ApJ 397,613

Hillenbrand L.A., Massey P., Strom S.E., Merrill K.M., 1993, AJ 106, 1906

Hoag A.A., Applequist L., 1965, ApJS 12, 215

Iben I., 1965, ApJ 141, 993 
Johnson H.L., 1966, ARA\&A 4, 193

Leizawitz D., 1988, Catalog of Open Clusters and Associated Interstellar Matter, NASA Ref. Pub. 1202, NASA Scientific and Technical Info. Div.

Lynga G., 1987, Catalog of Open Cluster Data (5th edition), available through NSSDC, Greenbelt, Maryland

Massey P., Garmany C.D., Silkey M., Degoia-Eastwood K., 1989, AJ 97, 107

Massey P., Thompson A.B., 1991, AJ 101, 1408

Mermilliod J.C., 1976, A\&A 53, 289

Mermilliod J.C., Mayor M., 1989, A\&A 219, 125

Ninkov Z., Bretz D.R., Easton R.L., Shure M., 1995, AJ 110, 2242
Nordstrom B., Andersen J., Andersen M.I., 1996, A\&A (in print)

Phelps R.L, Janes K.A., 1993, AJ 106, 1870

Sagar R., Richter T., 1991, A\&A 250, 324

Salpeter E.E., 1955, ApJ 121, 161

Savage B.D., Mathis J.S., 1979, Ann. Rev. Astron. Astrophys. 17,73

Scalo J.M., 1986, Fund. Cosmic Phys. 11, 1

Sears F.H., Joyner M.C., 1928, ApJ 67, 24

Taff L.G., 1974, AJ 79, 1280

Walker G.A.H., Hodge P., 1968, PASP 80, 290 